\title{
Novel Cruciform Structures as Model Compounds for Coordination Induced Single Molecule Switches
}

\author{
Sergio Grunder§a, Roman Huber ${ }^{\mathrm{b}}$, Songmei Wüb, Christian Schönenberger ${ }^{\mathrm{b}}$, Michel Calame ${ }^{\mathrm{b}}$, \\ and Marcel Mayor ${ }^{\star a c}$
}

§SCS Mettler-Toledo Award Winner (Oral Presentation)

\begin{abstract}
We have synthesized various molecular cruciforms consisting of two different crossing $\pi$-systems and comprising crosswise arranged thiol- and pyridine-anchor groups. With these model compounds we strive towards the investigation of a new switching concept based on the potential dependent coordination of pyridines to gold electrodes in an electrochemical set-up. Integration of these cruciform molecules between both electrodes of a mechanically controlled break junction in a liquid environment gave insight into their single molecule transport properties. These studies allowed individual transport characteristics to be assigned to the bar subunits of the cruciforms but also revealed the remaining experimental challenges to realize the suggested switching concept.
\end{abstract}

Keywords: Molecular electronics · Molecular switches · Single molecule conductance

\section{Introduction}

The ongoing feature size reduction in silicon-based electronic circuitry is facing physical and economical challenges, resulting in an increasing interest for alternative strategies. An individual molecule consisting of a few atoms is probably the smallest possible object that still provides the structural variety required to realize different electronic functions. The integration of single molecules into electronic devices - today referred to as 'molecular electronics' - can be considered as the ultimate size reduction in electronic circuitry. ${ }^{[1-5]}$ Rather driven by scientific curiosity than by the prospect of smaller and cheaper devices the interdisciplinary challenge of electronically contacting single molecules

\footnotetext{
${ }^{*}$ Correspondence: Prof. Dr. M. Mayor

Tel.: +41612671006

Fax: +41612671016

E-mail: marcel.mayor@unibas.ch

aDepartment of Chemistry

University of Basel

St. Johanns-Ring 19

$\mathrm{CH}-4056$ Basel

${ }^{\mathrm{b} D e p a r t m e n t}$ of Physics

University of Basel

Klingelbergstrasse 82

$\mathrm{CH}-4056$ Basel

Institute of Nanotechnology

Karlsruhe Institute of Technology

P. O. Box 3640

D-76021 Karlsruhe
}

was tackled by teams of synthetic chemists and experimental physicists. However, the minute size of molecules restricts the number of electrodes to two and consequently, applying the successful terminology of semiconductor electronics, also the variety of electronic functions realizable in the single molecule device is restricted. Nevertheless, monomolecular films and even single molecules have been addressed with electrodes, and the electronic response of an applied voltage can be measured.[6] Particularly appealing electronic functions based on a two terminal device are rectification and switching. ${ }^{[4]}$ To switch a molecule between two metastable states an external trigger is required. Various switching molecular systems responding to for example light, a chemical or electrochemical potential or a voltage pulse have already been reported. ${ }^{[7]}$ Interestingly, several switching devices were found empirically and their switching mechanisms remain the subject of current debate. ${ }^{[8]}$

During the last three years we followed a systematic approach. We proposed a new switching concept based on the interplay between the integrated molecule and the electrodes, designed and synthesized the required molecules and investigated their transport properties. ${ }^{[9,10]}$

The hypothesized switching concept relies on a structure consisting of two rigid-rod type $\pi$-systems which cross each other resulting in a molecular cruciform. Both $\pi$-systems as bars of the cruciform differ in their electronic transparency and in their terminal anchor groups to the elec- trodes. While one bar comprises terminal thiophenols which are known to bind strongly to gold electrodes over a large potential range, the second bar comprises terminal pyridines for which potential dependent coordination to gold substrates has been found in electrochemical STM experiments. ${ }^{[1-14]}$ As displayed in Fig. 1, we therefore suggested to control the coordination of the pyridine-terminated bar to the electrode by an electrochemical potential while the sulfur-terminated bar is keeping the cruciform positioned between both electrodes. In case both bars differ in their electronic transparencies, the number of bars bridging both electrodes should be reflected in the conductance of the junction, providing an electrochemically triggered switch.

In this paper we summarize our studies towards coordination induced single molecule switching. In particular the structural design and the synthesis of novel molecular cruciforms are presented together with single molecule transport investigations in a mechanically controlled break junction $(\mathrm{MCBJ})$ in a liquid environment. ${ }^{[9,10]}$

\section{Molecular Design}

To investigate the new switching mechanism, molecular cruciforms consisting of thiol and pyridine functionalized bars were required. Considerable knowledge concerning handling, synthesis and electronic transport investigation of terminally thiol functionalized rods had already been 

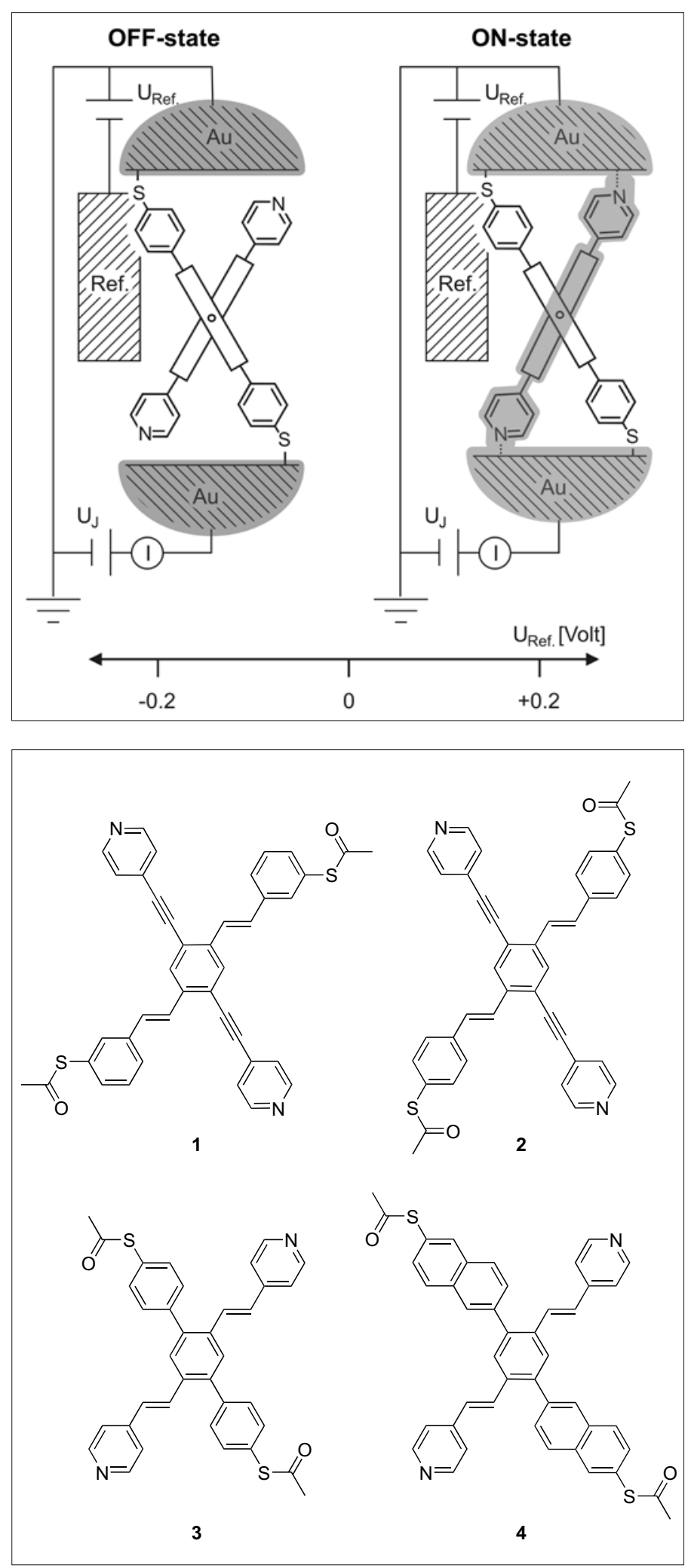

collected within the group. ${ }^{[15-19]}$ Due to the ideal strength of the gold-sulfur bond these rods remained moveable on the electrode surface and displayed rather poor conductivities, in part due to the tunnel barriers formed with the gold-sulfur bonds. ${ }^{[20,21]}$ Initially we thus expected an improved electronic coupling via pyridine subunits. However, transport studies based on model compounds synthesized within this project revealed the electronically superior cou-
Fig. 2. Cruciform structures 1 and 2 of the first generation and 3 and $\mathbf{4}$ of the second generation.

Fig. 1. Sketch of the proposed switching mechanism based on the electrochemical potential dependent coordination of the terminal pyridine subunits to the electrodes. In the OFFstate both electrodes are only bridged by the thiol functionalized bar which fixes the cruciform inside the junction. In the ONstate, the pyridinefunctionalized bar joins in providing an additional electronic transport channel.

via a terminal thiophenol compared to a terminal pyridine subunit, in spite of the shorter electrode-electrode distance in the later case. ${ }^{[10]}$

The first generation of cruciforms consisted of a sulfur-functionalized oligophenylenevinylene (OPV) fixation bar and a transversal pyridine-functionalized oligophenyleneethynylene (OPE) bar (Fig. $2)$. To disfavor the electronic transport through the fixation bar of the cruciform $\mathbf{1}$, the sulfur anchor groups were initially arranged in meta-positions. From earlier investigations it was known that the subtle structural variation of moving both anchor groups from the para-position to the metaposition reduces the electronic conductivity by more than one order of magnitude. ${ }^{[16]}$ Unfortunately, the electronic transparency of the meta-thiol functionalized OPV rod turned out to be outside the detectable range of the MCBJ set-up in a liquid environment. To enable at least initial investigations whether these rather bulky cruciforms can be integrated between both electrodes of a MCBJ, we also synthesized the corresponding cruciform 2 with sulfur anchor groups in para-position. Obviously, the resulting increased conductivity of the fixation bar contrasts to some extent the switching principle, which requires a considerably better conducting pyridinefunctionalized bar. The conductivity of the sulfur-bearing fixation bar needs to be low to suppress electron transport as it represents the OFF-state of the single molecule switch, but it has to be above the detection limit of the integration setup to observe the formation of the molecular junction. The superior conductivity of a thiol-functionalized rod compared with a pyridine functionalized analogue ${ }^{[10]}$ and the requirement of having the thiol groups in paraposition ${ }^{[9]}$ increased the molecular design challenges considerably. In order to tune the conductance values of the two rod substructures towards each other the position of the pyridine anchor group was changed to the OPV bar, as OPVs were found to be better conducting than OPEs. ${ }^{[22]}$ To keep an appropriate length ratio of the two bar substructures, the OPE system was substituted by an oligophenylene in the cruciform 3. Oligophenylene rods with substituents in ortho-positions to the bridging carbon atoms displayed reduced conductivities due to the orthogonal arrangement of the $\pi$-systems of both phenyl rings. ${ }^{[17]}$ Furthermore, to compensate the shortness of the oligophenylene bar of 3, naphthyl subunits were considered for the oligoaryl bar of 4.[10]

\section{Synthesis}

The target cruciforms 1-4 were synthesized with acetyl-protected sulfur groups. The masking of both thiophenols prevents these bifunctional building blocks from oxidative polymerization by disulfide formation and the acetyl group turned out to be easy cleavable in situ prior to the physical experiments. The synthetic strategy towards $\mathbf{1}$ and $\mathbf{2}$ relies on a Wittig reaction to assemble the OPV unit and a Sonogashira reaction to assemble the OPE bar. Using iodine leaving groups the palladium- and 


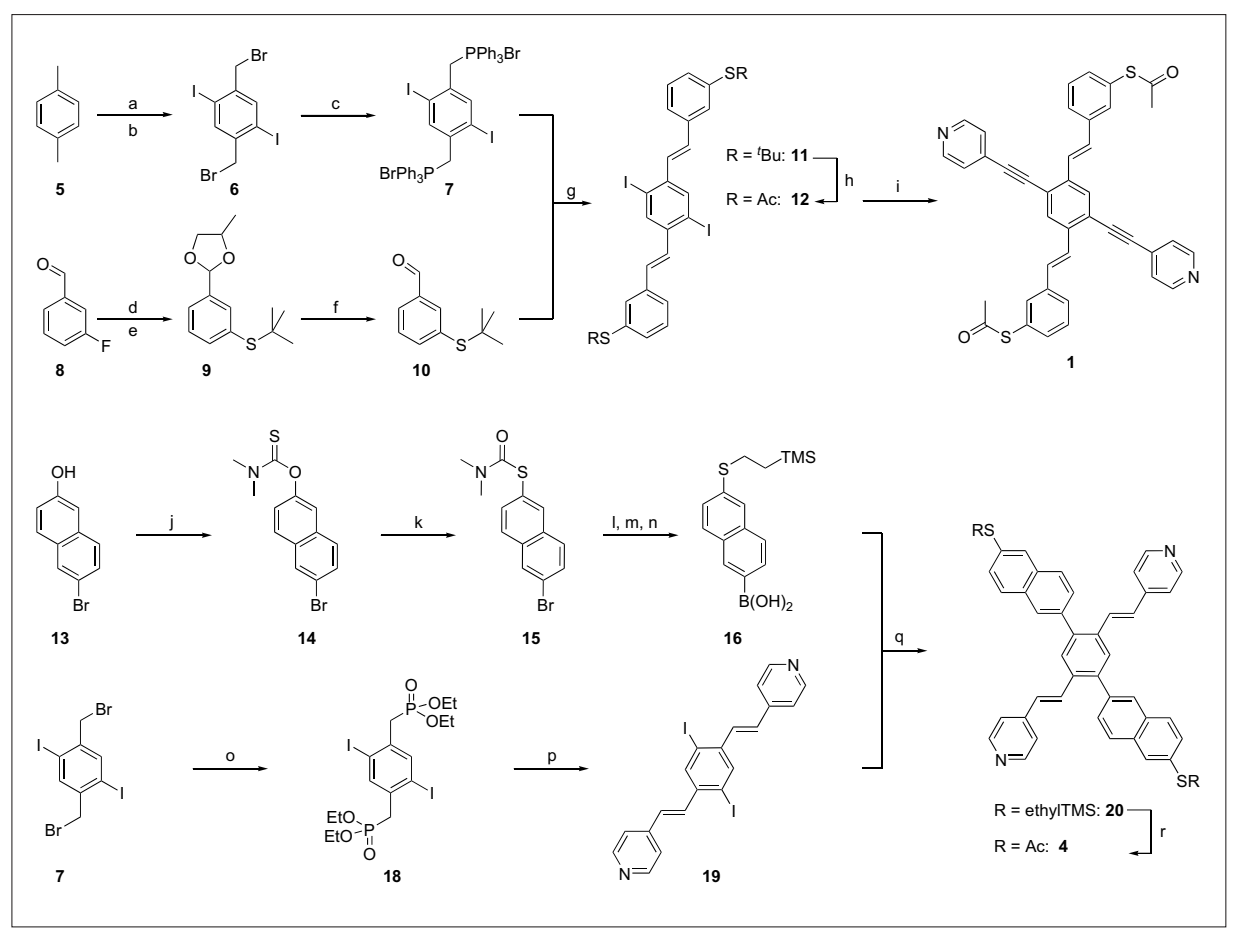

Scheme 1: a) I $, \mathrm{HIO}_{3}, \mathrm{AcOH}, \mathrm{H}_{2} \mathrm{SO}_{4}, 85^{\circ} \mathrm{C}, 79 \%$; b) $\mathrm{Br}_{2}, \mathrm{BrCH}_{2} \mathrm{CH}_{2} \mathrm{Br}, 135^{\circ} \mathrm{C}, 20 \%$, c) $\mathrm{PPh}_{3}, \mathrm{DMF}$ $85^{\circ} \mathrm{C}, 100 \%$; d) 1,2-propanediol, PTSA, toluene, $98{ }^{\circ} \mathrm{C}, 84 \%$; e) NaSt Bu, DMI, $150{ }^{\circ} \mathrm{C}, 66 \%$; f) $\mathrm{HCl}$, dioxane, r.t., $92 \%$; g) 1.) $\mathrm{NaOH}$, DCM, r.t., 2.) $\mathrm{I}_{2}$, toluene, $111{ }^{\circ} \mathrm{C}, 83 \%$; h) $\mathrm{BBr}_{3}, \mathrm{AcCl}$, toluene, r.t., 87\%; i) 4-ethynylpyridine, $\mathrm{Pd}\left(\mathrm{PPh}_{3}\right)_{4}$, Cul, $\mathrm{Pr}_{2} \mathrm{NH}, 45^{\circ} \mathrm{C}, 51 \%$; j) dimethylcarbamoylchloride, $\mathrm{NaH}$, DMF, $80^{\circ} \mathrm{C}$, quant.; k) phenylether, $259^{\circ} \mathrm{C}$, $89 \%$; I) $\mathrm{KOH}, \mathrm{MeOH}, 80^{\circ} \mathrm{C}, 97 \%$; m) vinyl trimethylsilane, AIBN, $100{ }^{\circ} \mathrm{C}, 96 \%$; n) $n$-BuLi, B(OiPr) $)_{3}$ THF, $-78{ }^{\circ} \mathrm{C} \rightarrow$ r.t., $76 \%$; o) P(OEt) $)_{3}, 150$ ${ }^{\circ} \mathrm{C}, 91 \%$; p) 4-pyridine carboxaldehyde, $\mathrm{NaH}$, THF, $50{ }^{\circ} \mathrm{C}, 67 \%$; q) $\mathrm{Pd}\left(\mathrm{PPh}_{3}\right)_{4}, \mathrm{~K}_{2} \mathrm{CO}_{3}$, toluene, ethanol, $80^{\circ} \mathrm{C}, 85 \%$; r) TBAF, AcCl, THF, r.t., $58 \%$.

copper-catalyzed Sonogashira coupling reaction proceeds at moderate temperatures $\left(45^{\circ} \mathrm{C}\right)$ allowing the presence of the acetyl-protected thiophenols, which were thus introduced in advance. The OPV substructure in target structure $\mathbf{3}$ and $\mathbf{4}$ were assembled using a Horner-WadsworthEmmons (HWE) reaction while the oligoaryl bar was obtained by a Suzuki reaction. As the acetyl protection groups would not survive the basic conditions of the Suzuki reaction, they were introduced in the last step. Ethyl-trimethylsilane (ethyl-TMS) protection groups were used to mask the reactivity of the thiophenols during the assembly of the cruciforms instead. Due to the limited space and the modular and repetitive nature of their assembly, only the synthesis of the cruciforms $\mathbf{1}$ and $\mathbf{4}$ as representative examples will be summarized in the following (Scheme 1).

Starting with para-xylene (5), aromatic iodination followed by bromination of the methyl groups provided the suitably functionalized compound $\mathbf{6}$ as precursor of the central phenyl ring. Substitution of the benzylic bromides with triphenylphosphine gave the bis-phosphonium salt 7 as starting material of the Wittig reaction. The aldehyde $\mathbf{1 0}$ as second precursor of the Wittig reaction was obtained from commercially available 3-fluorobenzaldehyde (8). After protecting the aldehyde $\mathbf{8}$ as acetal the flu- orine atom was substituted by tert-butylthiolate. The aldehyde $\mathbf{1 0}$ was obtained by cleaving the acetal under acidic conditions. The aldehyde $\mathbf{1 0}$ and the bis-phosphonium salt 7 formed together the desired ethenylbridged aryl structure as a mixture of the $E / Z$-isomers by treatment with aqueous sodium hydroxide. A subsequent addition/ elimination sequence with iodine in refluxing toluene provided the $E / E$-isomer $\mathbf{1 3}$ in $83 \%$ yield. Transprotection of the terminal sulfur anchor groups was achieved by treatment with boron tribromide followed by acetyl chloride to yield in the acetylprotected building block 12. To assemble the target structure $\mathbf{1}$, both iodines of $\mathbf{1 2}$ were substituted by 4-ethynylpyridine in a Sonogashira reaction. Purification by precipitation and recrystallization provided the cruciform $\mathbf{1}$ as a yellow solid in $51 \%$ yield. A similar sequence of reaction steps provided the cruciform 2. Synthetic protocols together with the analytical data of all intermediates and target structures are reported in ref. [9].

The bifunctional naphthalene building block 16 having a masked thiol group and a boronic acid in 2- and 6-position respectively was required for the assembly of the cruciform 4. As a suitable commercial precursor 6-bromonaphthalen-2-ol (13) was considered. While the bromine substituent is ideally suited to introduce a boronic acid the phenolic hydroxy group allows the introduction of a sulfur substituent via a Newman-Kwart rearrangement (NKR). [23] Thus $\mathbf{1 3}$ was treated with dimethylcarbamoylchloride to provide the $O$-thiocarbamate $\mathbf{1 4}$. The thermally activated NKR was triggered by heating $\mathbf{1 4}$ to $259{ }^{\circ} \mathrm{C}$ to give the $S$-thiocarbamate $\mathbf{1 5}$ in good yields. Hydrolysis of the thiocarbamate $\mathbf{1 5}$ and subsequent protection of the phenolic thiol in a radical addition reaction with vinyl-trimethylsilane led to the ethylTMS protected building block. After treatment of the obtained bromide with $n$-BuLi and triisopropylborate the boronic acid $\mathbf{1 6}$ was obtained after aqueous workup. In an Arbusov reaction the bromides of the central building block 7 were substituted with triethylphosphite to give the phosphonate 18. In a HWE reaction 18 and 4-pyridinecarboxaldehyde provided exclusively the desired $E / E$-isomer 19 as first bar of the cruciform 4 comprising two iodine leaving groups. The cruciform was assembled in a Suzuki reaction of $\mathbf{1 9}$ with the boronic acid 16. Finally, the ethyl-TMS protected terminal sulfur anchor groups were liberated with fluoride ions and de novo protected in situ by treatment with an excess acetyl chloride. Thereby also the terminal pyridine groups were acetylated. However, the acetylated pyridines were hydrolyzed selectively in an ethanol chloroform mixture providing the target structure 4 in 58\% yield as a yellow solid. A comprehensive discussion of the synthesis together with analytical data of all intermediates and target structures are reported in ref. [10].

\section{Single Molecule Transport Studies}

The electronic transport properties of the novel cruciform structures 1-4 were investigated on a single molecule level in a MCBJ setup in a liquid environment. ${ }^{[9,10,24]}$ The MCBJ relies on a microfabricated gold structure (B in Fig. 3) which is bent in a three-point bending mechanism (A in Fig. 3) such that, at one point, the gold structure breaks apart to form two atomically sharp electrodes. While adjusting the pushing rod distance $\mathrm{z}$, the distance between the two electrodes can be adjusted with a resolution better than an Ångstrom, making the MCBJ a well-suited tool to investigate single molecules. Details concerning the experimental setup can be found in refs $[9,10,22,24]$. To get information about the junction, the conductance between the two electrodes was examined as a function of the pushing rod distance $\mathrm{z}$ resulting in typical conductance traces $(\mathrm{C}$ in Fig. 3). After breaking the last gold contact, an abrupt down-jump followed by an exponential decay curve is observed when only solvent molecules are present. How- 

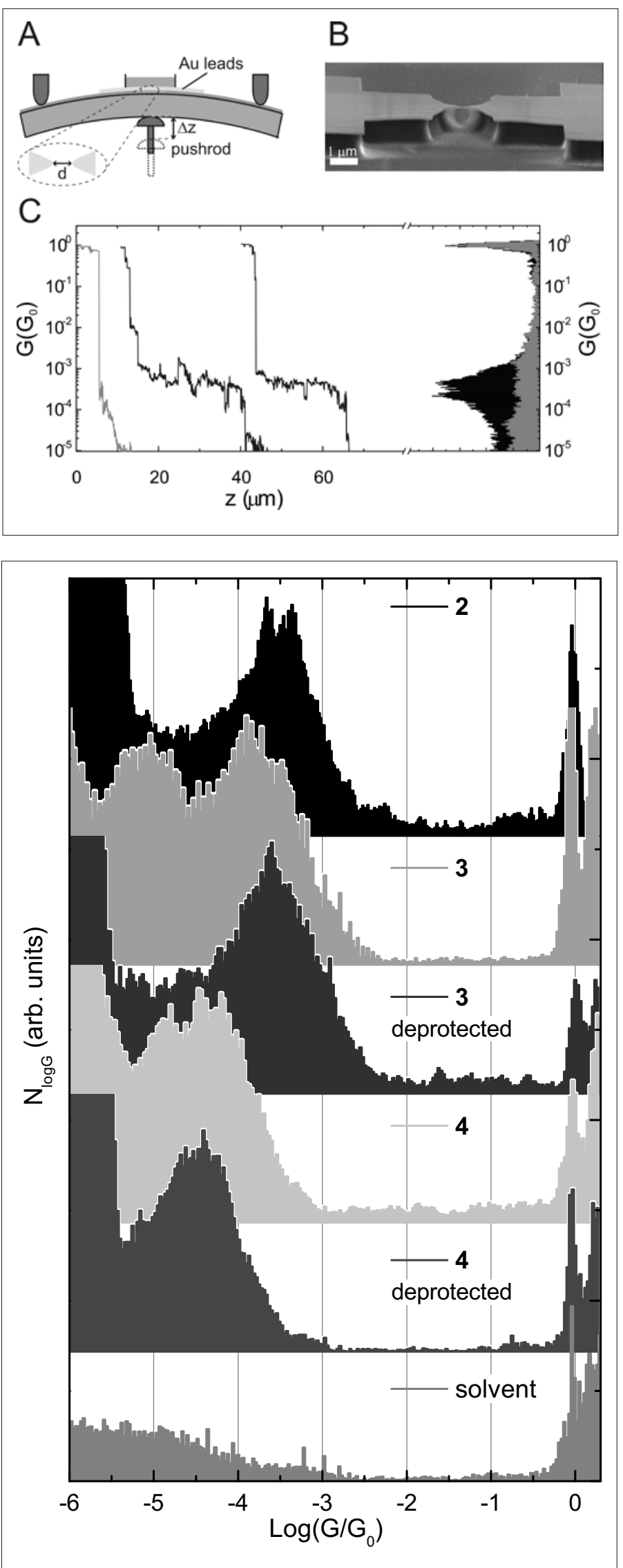

ever, if molecules bearing suitable anchor groups to bridge both electrodes are exposed to the setup, the conductance traces show plateaus which are attributed to the formation of molecule junctions. To balance fluctuations in the conductance trac- es of these sensitive junctions, numerous junctions were analyzed statistically.[25] Therefore hundreds of conductance traces were measured and collected in histograms showing peaks at the positions of the most abundant conductance plateaus.
Fig. 4. LogG histograms of the cruciforms 2-4 and of the solvent mixture THF/mesitylene (1:4). For the cruciforms $\mathbf{3}$ and $\mathbf{4}$ histograms with and without the addition of a deprotection agent

(TBAOH) are displayed.

Fig. 3. A) Schematic representation of the MCBJ setup with the underlying three point bending mechanism. B) Scanning electron microscopy (SEM) mage of the microfabricated gold structure. C) The conductance between inductance traces. When only solvent molecules are present (grey 作 A statistical analysis of the current traces is performed while forming histograms.

When only solvent molecules are present, no peak in the $\log G$ histogram is observed besides the peak at $1 \mathrm{G}_{0}$, which is attributed to the conductance of the last gold contact (77.5 $\mu \mathrm{S})$. To investigate the cruciforms 1-4, these were dissolved in tetrahydrofuran (THF)/mesitylene (1:4) and applied to the MCBJ setup with or without deprotection with tetrabutylammonium hydroxide (TBAOH). Typical histograms obtained for the cruciforms 2-4 are displayed in Fig. 4. Surprisingly, no molecular signature was observed in the histogram of the cruciform 1. Presumably the considerable loss of conjugation due to its sulfur anchor groups in meta-positions resulted in transport currents below the detection threshold of the setup. To strengthen this hypothesis $\mathbf{2}$ was investigated as control structure with increased conductivity through the sulfurterminated bar. And indeed, a peak in the $\log \mathrm{G}$ histogram at $2.2 \times 10^{-4} \mathrm{G}_{0}$ was observed pointing at single molecules bridging both gold electrodes via their thiol anchor groups. ${ }^{[9]}$ While the single molecule traces demonstrate that even rather bulky molecules like the cruciform $\mathbf{2}$ can form molecular junctions, the superior conductance of the sulfur-functionalized bar did not allow detection of conduction alterations arising from the coordination of the pyridine bar. To improve the transport characteristics the pyridines were placed on the OPV bar in $\mathbf{3}$ and $\mathbf{4}$. Interestingly, two peaks were observed in the $\log \mathrm{G}$ histograms of both compounds $\mathbf{3}$ and $\mathbf{4}$ when no deprotection agent was added. Comparison of the electronic signatures of the cruciforms 3 and $\mathbf{4}$ with that of the parent bar structures allowed the peaks to be attributed. While for both cases the higher conductance peak was ascribed to the sulfur-functionalized fixation bar, the lower conductance peak arises from the pyridinefunctionalized bar. ${ }^{[10]}$ The observation of two peaks in the histograms is assumed to arise from part of the molecules binding via the pyridine anchor groups and part of the molecules binding via spontaneously deprotected thiol anchor groups. If these cruciforms were deprotected prior to exposition to the setup offering the free thiol for binding, one distinct peak is observed in the histogram arising from the sulfurterminated bar dominating the conduc- 
tance characteristics. The conductance of the pyridine-functionalized rod substructure moved closer to the conductance of the fixation bar by elongating the sulfurterminated bar from $\mathbf{3}$ to $\mathbf{4}$, but still remains below the threshold of the fixation bar.

\section{Summary and Conclusion}

Our efforts towards a new switching concept based on the interplay between molecule and electrode are summarized. The proposed switching mechanism relies on the electrochemical potential dependent coordination of pyridine anchor groups to gold electrodes. Different cruciform structures consisting of a terminally sulfurfunctionalized fixation bar and a switching bar comprising terminal pyridine groups were synthesized and fully characterized. Single molecule transport properties of these cruciforms were investigated in a mechanically controlled break junction (MCBJ) setup in a liquid environment.

A full in situ electrochemical operation of the cruciform switch remains to be demonstrated. Also, the inspection of the transport properties of the cruciforms together with model compounds consisting of single bars as their parent substructures exhibit that further tuning of the electronic transport properties is required. Nevertheless, it was possible to integrate the rather bulky cruciform molecules bearing the required functions for the proposed coordination-induced switching mechanism on a single molecule level. Furthermore, we were able to demonstrate that both bars of the cruciforms $\mathbf{3}$ and $\mathbf{4}$ were able to bridge the gap between both electrodes.

To realize the proposed switching mechanism we are currently working on two issues. While the physicists are improving their setup to reduce the detection threshold the chemists are improving the molecular design to further improve the electronic transport through the pyridinefunctionalized rod with respect to the sulfur-functionalized fixation rod.

\section{Acknowledgment}

Financial support by the Swiss National Science Foundation (SNSF), the NCCR nanoscale science and the University of Basel is gratefully acknowledged.

Received: December 24, 2009
[1] C. Joachim, J. K. Gimzewski, A. Aviram, Nature 2000, 408, 541.

[2] J. R. Heath, M. A. Ratner, Phys. Today 2003, $56,43$.

[3] N. J. Tao, Nat. Nanotechnol. 2006, 1, 173 .

[4] R. L. Carroll, C. B. Gorman, Angew. Chem., Int. Ed. 2002, 41, 4378.

[5] N. Weibel, S. Grunder, M. Mayor, Org. Biomol. Chem. 2007, 5, 2343.

[6] H. Haick, D. Cahen, Prog. Surf. Sci. 2008, 83, 217.

[7] 'Molecular Switches', Ed. B. L. Feringa, WileyVCH, Weinheim, 2001.

[8] E. Lörtscher, J. W. Ciszek, J. Tour, H. Riel, Small 2006, 2, 973.

[9] S. Grunder, R. Huber, V. Horhoiu, M. T. González, C. Schönenberger, M. Calame, M. Mayor, J. Org. Chem. 2007, 72, 8337.

[10] S. Grunder, R. Huber, S. Wu, C. Schönenberger, M. Calame, M. Mayor, Eur. J. Org. Chem. 2010, $5,833$.

[11] B. Xu, N. J. Tao, Science 2003, 301, 1221.

[12] D. Mayer, T. Dretschkow, K. Ataka, T. Wandlowski, J. Electroanal. Chem. 2002, 20, 524.

[13] X. Li, J. Hihath, F. Chen, T. Masuda, L. Zang, N. J. Tao, J. Am. Chem. Soc. 2007, 129, 11535.

[14] F. Cunha, N. J. Tao, X. W. Wang, Q. Jin, B Duong, J. D'Agnese, Langmuir 1996, 12, 6410.

[15] J. Reichert, R. Ochs, D. Beckmann, H. B. Weber, M. Mayor, H. von Löhneysen, Phys. Rev. Lett. 2002, 88, 176804/1.

[16] M. Mayor, B. Weber Heiko, J. Reichert, M. Elbing, C. von Hänisch, D. Beckmann, M. Fischer, Angew. Chem., Int. Ed. 2003, 42, 5834.

[17] E. Lörtscher, M. Elbing, M. Tschudy, C. von Hänisch, H. B. Weber, M. Mayor, H. Riel, ChemPhysChem 2008, 9, 2252.

[18] M. Elbing, R. Ochs, M. Koentopp, M. Fischer, C. von Hänisch, F. Weigend, F. Evers, H. B. Weber, M. Mayor, Proc. Natl. Acad. Sci. USA 2005, 102, 8815.

[19] M. Mayor, C. von Hänisch, B. Weber Heiko, J. Reichert, D. Beckmann, Angew. Chem., Int. Ed. 2002, 41, 1183 .

[20] K. W. Hipps, Science 2001, 294, 536.

[21] F. Chen, X. Li, J. Hihath, Z. Huang, N. J. Tao, J. Am. Chem. Soc. 2006, 128, 15874

[22] R. Huber, M. T. González, S. Wu, M. Langer, S. Grunder, V. Horhoiu, M. Mayor, M. R. Bryce, C. Wang, R. Jitchati, C. Schönenberger, M. Calame, J. Am. Chem. Soc. 2008, 130, 1080.

[23] G. C. Lloyd-Jones, J. D. Moseley, J. S. Renny, Synthesis 2008, 661.

[24] L. Grüter, M. T. González, R. Huber, M. Calame, C. Schönenberger, Small 2005, 1, 1067.

[25] M. T. González, S. Wu, R. Huber, S. J. van der Molen, C. Schönenberger, M. Calame, Nano Lett. 2006, 6, 2238. 